\title{
Trehalose Inhibits Oxygen Glucose Deprivation Induced Autophagic Death in Dopaminergic SH-SY5Y Cells via Mitigation of Glycolysis Dysfunction and Inhibiting Activated Protein Kinase Activation
}

\author{
BENXUAN WANG ${ }^{1,3}$, YANG JIAO ${ }^{1}$, DAN XU ${ }^{1}$, QINGMEI JIANG ${ }^{1}$, YUHANG REN ${ }^{1}$, FENGJIANG CHEN² AND CHANGHAO YIN ${ }^{1 *}$ \\ Department of Neurology, Hongqi Hospital Affiliated to Mudanjiang Medical University, ${ }^{1}$ Heilongjiang Key Laboratory of Ischemic \\ Stroke Prevention and Treatment, Mudanjiang, Heilongjiang Province 157011, ${ }^{2}$ Department of Physiology, Heilongjiang Traditional \\ Chinese Medicine University Jiamusi college, Jiamusi, Heilongjiang Province 154007, ${ }^{3}$ Department of Neurology, Jinnan Hospital, \\ Tianjin 300350, China
}

Wang et al.: Trehalose Inhibits Autophagic Death in Dopaminergic SH-SY5Y Cells

\begin{abstract}
The goal of this study was to explore the regulatory mechanism of trehalose on the autophagy death of SHSY5Y cells induced by oxygen and sugar deprivation from the aspect of glycolysis. Human SH-SY5Y cells were divided into three groups includes control group (control), model group (oxygen and sugar deprivation) and experimental group (oxygen and sugar deprivation+trehalose). The proliferation activity, mortality of SH-SY5Y cells, adenosine triphosphate and pyruvic acid were measured. Western blot was carried out to detect the expression levels of autophagic-related proteins (Unc-51 like autophagy activating kinase 1, beclin 1, autophagy related 5, microtubule-associated protein 1A/1B-light chain 3, ubiquitin-binding protein p62), glycolysis-related proteins (hexokinase 2, phosphofructokinase, pyruvate kinase M2) and activated protein kinase signalling pathway proteins. Compared with the control group, the autophagy level of the oxygen and sugar deprivation group was increased in a time-dependent manner $(\mathbf{p}<0.05)$. Cell mortality was increased $(p<0.05)$ and the expressions of hexokinase 2 , phosphofructokinase, pyruvate kinase M2 were decreased $(p<0.05)$. The expression levels of activated protein kinase signalling pathway related proteins was upregulated $(p<0.05)$. On the contrary, the cell death rate and autophagy level of the experimental group were significantly lower than that of the model group. The protein levels of hexokinase 2, phosphofructokinase, pyruvate kinase M2 were up-regulated and the difference was statistically significant $(p<0.05)$. Trehalose can reduce the damage of SH-SY5Y cells caused by oxygen and sugar deprivation. The mechanism may be related to the improvement of glycolysis dysfunction and alleviation of autophagy over activation of activated protein kinase.
\end{abstract}

Key words: Glycolysis, trehalose, autophagy, activated protein kinase signalling pathway

Ischemic cerebrovascular disease is a common neurological disease with an incidence of approximately $16 \%$ of the global population ${ }^{[1]}$. Ischemic cerebrovascular disease refers to cerebral blood supply disorders that occur on the basis of cerebrovascular lesions or hemodynamic disorders, resulting in a series of neurological deficits. Its pathophysiological mechanisms involve various biological events such as neuronal apoptosis, oxidative stress, endoplasmic reticulum stress and inflammatory cascade ${ }^{[2-5]}$. Although the success rate of endovascular treatment has been significantly improved in recent years, there is still a lack of effective treatment.
Glycolysis refers to the process by which glucose is decomposed into pyruvate in the cytoplasm and produces large amounts of lactate ${ }^{[6]}$. In the early stage of ischemia and hypoxia, cells will increase the energy supply to the ischemic area, especially the functional area of the ischemic penumbra, by regulating glycolysis. Autophagy is a process that relies on lysosomal pathways for the degradation of cytoplasmic proteins and organelles and plays an important role in the pathology of brain injury such as hypoxia ischemia $^{[3]}$. Autophagy is activated when glycolysis is impaired and becomes the driving force of Adenosine Triphosphate (ATP) oxidative phosphorylation. In cells

*Address for correspondence

E-mail: yinchanghao79623@163.com 
that are highly dependent on glycolysis to provide energy, adenylate accumulation (increased Adenosine Monophosphate (AMP)/ATP ratio) and AMP-Activated Protein Kinase (AMPK) pathway activation are usually the main reasons for triggering autophagy ${ }^{[6]}$. Glycolytic dysfunction has been found to cause energy failure and further trigger autophagy, ultimately leading to autophagic cell death ${ }^{[7-10]}$. Therefore, it is important to improve cellular glucose metabolism and energy supply to reduce neuronal injury for cerebral ischemic damage ${ }^{[4]}$.

Trehalose is a safe and stable disaccharide composed of two molecules of glucose ${ }^{[11]}$ which is both an energy source and an important stress protectant ${ }^{[12]}$ and it can rescue cell death by inhibiting the over activation of autophagy, oxidative stress, endoplasmic reticulum stress and acting as a chaperone in the SH-SY5Y cell oxygen-glucose deprivation model ${ }^{[13-15]}$. In this study, we established a SH-SY5Y oxygen-glucose deprivation model to study the regulatory role of glycolysis in autophagic death and the intervention mechanism of trehalose, providing potential therapeutic drugs for the treatment of ischemic cerebrovascular disease.

\section{MATERIALS AND METHODS}

\section{Establishment of cell culture and Oxygen Glucose Deprivation (OGD) models:}

SH-SY5Y cells were obtained from Shanghai Institute of Cell Biology, Chinese Academy of Sciences. Cell cultures were maintained in Dulbecco's Modified Eagle Medium (DMEM) containing $10 \%$ fetal bovine serum; penicillin $(100 \mathrm{U} / \mathrm{ml})$ and streptomycin $(100$ $\mu \mathrm{g} / \mathrm{ml}$ ) were placed in an incubator at $37^{\circ}$ and $5 \%$ Carbon Dioxide $\left(\mathrm{CO}_{2}\right)$ with medium changes twice a week. Logarithmically growing cells were seeded into 96-well plates $\left(5-6 \times 10^{3} /\right.$ well $)$ and the next day, the adherent cells were replaced with sugar-free medium, placed in a hypoxic culture device and filled with a mixture of $5 \% \mathrm{CO}_{2}$ and $95 \%$ nitrogen and cultured in a constant temperature incubator at $37^{\circ}$ for $24 \mathrm{~h}$. After $24 \mathrm{~h}$, the drug is successively added and processed with OGD. The cell death rate is detected according to the Lactate Dehydrogenase (LDH) kit (Biyuntian, China). The absorbance value of the detected sample at 490 $\mathrm{nm}$ and the cell death rate percentage $=$ (sample-control/ $\mathrm{A}_{\max }$-control) $\times 100 \%$.

\section{Lentiviral Light Chain 3 (LC3) transfection into SH-SY5Y cells:}

Select SH-SY5Y cells with good selection status and strictly follow the reagent instructions to set the density as $(3-5) \times 10^{4}$ per $\mathrm{ml}$ cells were seeded in $2.5 \mathrm{~cm}$ culture dishes and placed in a cell incubator for routine culture. Replace the culture medium by referring to the volume in the instructions for use and add the corresponding infection enhancement solution, place the cells in $37^{\circ}$ incubator for cultivation for $12 \sim 16 \mathrm{~h}$ and then perform routine culture.

\section{ATP and pyruvate determination:}

ATP production and pyruvate production were measured according to the instructions of the ATP kit and the instructions of the pyruvate content kit, respectively. ATP generation amount of cells $(\mu \mathrm{mol} /$ $\mathrm{g})=($ assay well-control well)/(standard well-blank well $) \times$ standard concentration $\left(1 \times 10^{3}\right) /$ sample protein concentration $(\mathrm{g} / \mathrm{l})$. Pyruvate generation amount $(\mu \mathrm{mol} /$ $\mathrm{mg})=$ standard concentration $\times($ measured value-blank Optical Density (OD) value)/(standard OD valueblank OD value) $\div$ protein concentration of sample to be tested. The detection of ATP production and pyruvate production was statistically analysed and plotted with Graphpad Prism 6.0.

\section{Detection of related protein expression in cells of each group by Western blot:}

The supernatant of cells to be detected was discarded, washed twice with precooled phosphate-buffered saline and placed on an ice plate, lysed with cell lysate for $30 \mathrm{~min}$ and protein quantification was performed by Bicinchoninic Acid (BCA) assay. Each group of samples was separated on $10 \%$ sodium dodecylsulfate polyacrylamide gel electrophoresis gel and then transferred to polyvinylidene fluoride membrane (Millipore USA). $5 \%$ skimmed milk powder was blocked at room temperature for $1 \mathrm{~h}$ and then incubated at $4^{\circ}$ for primary antibody. The specific amount of primary antibody used was as follows: Anti-Unc-51 Like Autophagy Activating Kinase 1 (anti-ULK1) (CST 8054, 1:1000, USA), anti-phospho-ULK1 (anti-pULK1) (CST 5869, 1:1000, USA), anti-AMP-Activated Protein Kinase (anti-AMPK) (CST 9158, 1:1000, USA) and anti-phospho-AMPK (anti-p-AMPK) (CST 50081, 1:1000, USA), anti-LC3 (Sigma SAB1305638, 1:1000, USA), anti-beclin 1 (CST 3495, 1:1000, USA), antiAutophagy Related 5 (ATG5) (CST 12994, 1:1000, USA), anti-ubiquitin-binding protein p62 (Abcam ab109012, 1:1000, USA), anti-Hexokinase 2 (HKII) (Abcam ab209847, 1:1000, USA), anti-Pyruvate Kinase M2 (PKM2) (Abcam ab85555, ab85555, USA) 1:1000 in the US), anti-Phosphofructokinase (PFKP) (CST company 12746, 1:1000 in the US); the next day, the secondary antibody was incubated at room 
temperature for $2 \mathrm{~h}$. The enhanced chemiluminescence kit was luminescent developed in the dark room and the image was obtained by the gel imaging system. The bands were densitometrically analyzed with ImageJ.

\section{Statistical analysis:}

All statistical analyses were performed using Statistical Package for the Social Sciences (SPSS) 22.0 software and plotted with the software Graphpad Prism 6.0 and the experimental data were expressed as mean \pm standard deviation. Statistical comparisons were performed using one-way Analysis of Variance (ANOVA). In some cases, t-tests were used to compare means between two groups. Data are presented as Standard Error of the Mean (SEM). Differences were considered statistically significant at $\mathrm{p}<0.05$.

\section{RESULTS AND DISCUSSION}

LDH release assay showed that SH-SY5Y cells had increased cell mortality under OGD (fig. 1A) and the results of Western blot showed that beclin1, ATG5 and LC3 as autophagy marker proteins had increased protein expression and decreased $\mathrm{p} 62$ protein expression under OGD (fig. 1B). We found numerous red and green fluorescent spots in cells treated with OGD for $24 \mathrm{~h}$ and yellow fluorescent spots in merged images compared to controls (fig. 1C). This suggests that OGD promotes LC3 formation. Points in the merged image with only red but no green fluorescence were also detected (arrow). Key evidence for autophagic flux, because during autophagy-lysosome fusion, sensGFP-LC3 is sensitive to the lysosomal acidic microenvironment and undergoes degradation. Thus, we demonstrate that autophagic flux is activated in cells exposed to OGD.

LDH release assay showed that pretreatment with autophagy inhibitor 3-Methyladenine (3-MA) (5 $\mathrm{mmol} / \mathrm{l})$, inhibited OGD-induced SH-SY5Y cell death (fig. 2A) and after ATG5 small interfering Ribonucleic

Acid (siRNA) knockdown, SH-SY5Y cell death rate was decreased compared with the OGD group (fig. 2B). The results of Western blot assay showed that 3MA pretreatment reversed beclin1, ATG5, LC3 and p62 under the condition of OGD (fig. 2C) and ATG5 siRNA knockdown reversed the expression of autophagy related proteins (fig. 2D).

Glycolysis acts as one of the major energy production pathways during ischemia and hypoxia and its dysfunction leads to a decrease in the energy end products ATP and pyruvate (fig. 3A and fig. 3B). Western blot results suggested that HKII, a key ratelimiting enzyme of glycolysis and PFKP and PKM2 protein levels were down-regulated in a time-dependent manner with the duration of OGD exposure (fig. 3C); LDH release assay: Pre-Autophagosomal Structure (PAS) simultaneously rescued SH-SY5Y cell death rate (fig. 3D). PAS Changes in autophagy-related proteins were reversed after pretreatment (fig. 3E).

The results of LDH assay indicated that compound C $(20 \mu \mathrm{mol} / \mathrm{l})$, an AMPK inhibitor, alleviated OGD induced cell death in SH-SY5Y cells (fig. 4A); AMPK, p-AMPK, ULK1 and p-ULK1 were activated in a timedependent manner under OGD (fig. 4B); compound $\mathrm{C}$ not only inhibited the protein expression of AMPK and p-AMPK, but also inhibited the expression of autophagy related proteins (fig. 4C and fig. 4D).

Trehalose significantly reduced OGD-induced cell death in SH-SY5Y cells (fig. 5A). Western blot results: Trehalose reversed the OGD-induced up-regulation of LC3, beclin1, ATG5 and down-regulation of p62 (fig. 5B), trehalose alleviated the OGD-induced upregulation of protein expression of AMPK, p-AMPK (fig. 5C). Trehalose upregulated the protein expression of HKII, PFKP and PKM2 which are key rate limiting enzymes of glycolysis (fig. 5D).

A

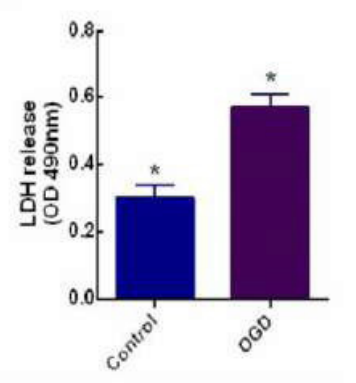

B

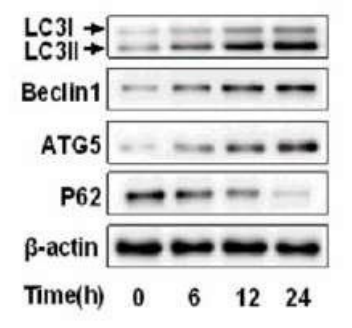

C

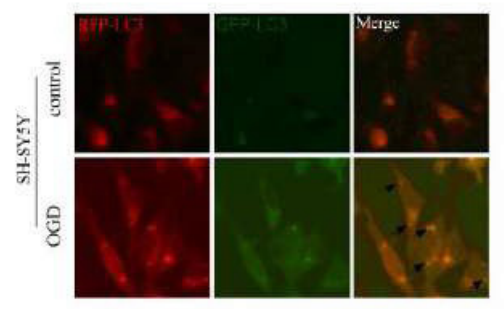

Fig. 1: OGD triggered SH-SY5Y cell death accompanied by activation of cellular autophagy levels, (A) LDH release assay indicated that OGD triggered SH-SY5Y cell death; (B) Western blot analysis showed that OGD induced the activation of autophagy in SH-SY5Y cells and (C) StubRFP-sens-GFP-LC3 lentivirus was transfected into SH-SY5Y cells and autophagy flux was changed in SH-SY5Y cells Note: ${ }^{*} \mathbf{p}<\mathbf{0 . 0 5}$ compared with control group 


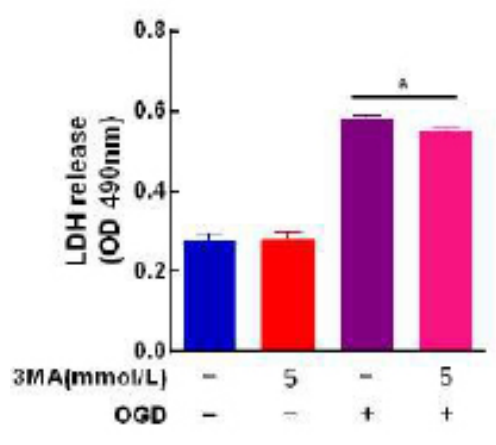

C

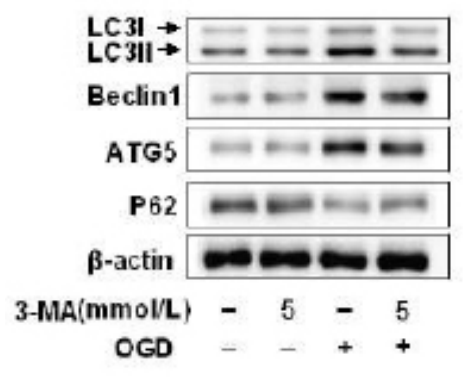



D

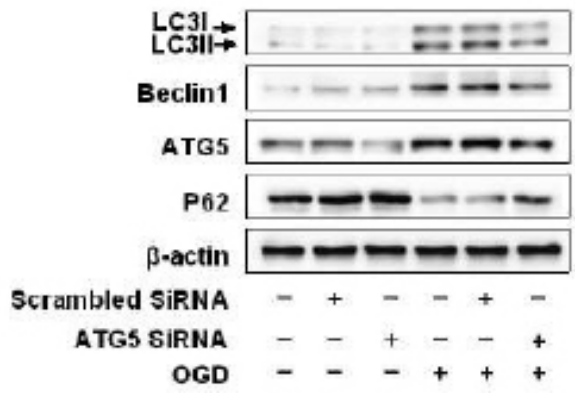

Fig. 2: OGD induced autophagic death of SH-SY5Y cells, (A) LDH release assay showed that 3MA inhibited OGD-induced cell death in SH-SY5Y cells; (B) LDH release assay showed that cell mortality was reduced after ATG5 siRNA knockdown; (C) Western blot assay results showed that 3MA pre-treatment reversed the protein expression of beclin 1, ATG5, LC3 and p62 proteins in response to OGD and (D) Western blot analysis showed that ATG5 siRNA knockdown reversed the expression of autophagy related proteins Note: * $\mathbf{p}<0.05$ compared with the control group

A



D

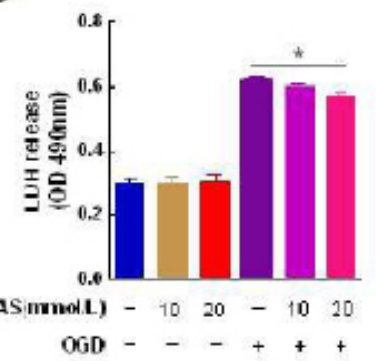

B

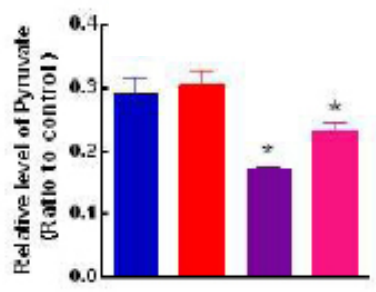

E



C



Fig. 3: OGD causes glycolysis dysfunction and autophagic death, (A, B) ATP and pyruvate detection results suggested that OGD led to a decrease in cellular ATP and pyruvate production and trehalose pre-treatment could increase cellular ATP and pyruvate production; (C) Western blot results showed that OGD induced a down-regulation of the expression of HKII, PFKP and PKM2, key rate-limiting enzymes of glycolysis; (D) LDH release assay showed that PAS pre-treatment alleviated OGD-induced SH-SY5Y cell death and (E) Western blot results showed that PAS pre-treatment reversed OGD-induced autophagy-related protein expression, $(\square)$ Control; $(\square)$ trehalose; ( $\square$ ) OGD and ( $\square$ ) OGD+trehalose

Note: * $\mathbf{p}<0.05$ compared with the control group 
A



C

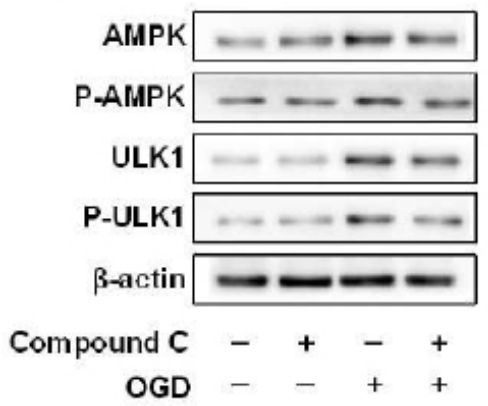

B



D

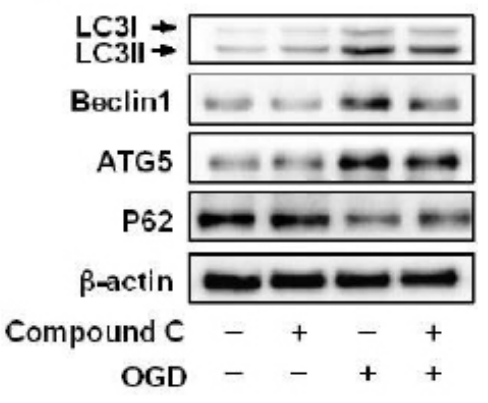

Fig. 4: OGD induced autophagic death of SH-SY5Y cells by activating AMPK, (A) LDH release assay showed that compound C pre-treatment alleviated OGD-induced cell death in SH-SY5Y cells; (B) Western blot analysis showed that OGD can be activated in a time-dependent manner AMPK signaling pathway also induced up-regulation of ULK1 and p-ULK1 protein expression; (C) Pre-treatment with compound C inhibited AMPK, p-AMPK, ULK1 and p-ULK1 protein expression and (D) Compound C

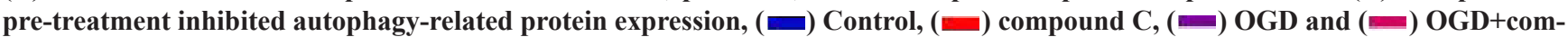
pound $\mathrm{C}$

Note: * $\mathbf{p}<0.05$ compared with the control group

A

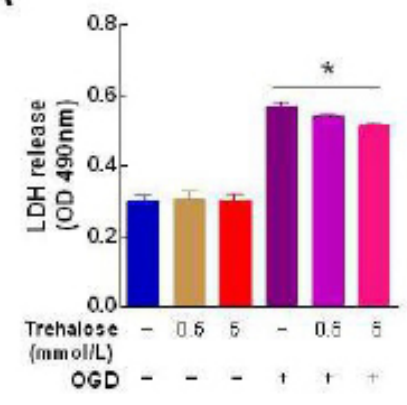

C

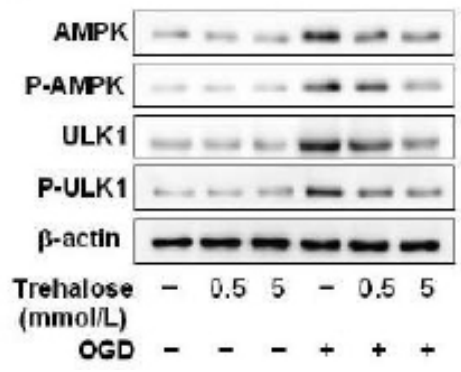

B



D

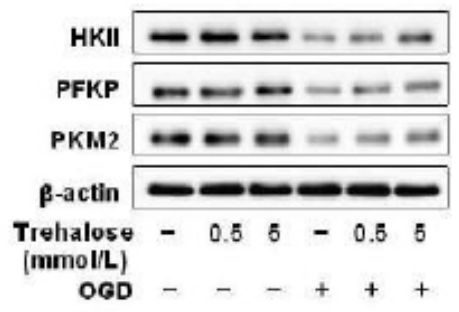

Fig. 5: Trehalose can alleviate cellular autophagic death by improving glycolytic dysfunction to increase cellular energy supply, (A) LDH release assay showed that trehalose pre-treatment alleviated OGD-induced cell death in SH-SY5Y cells; (B) Western blot analysis showed that trehalose alleviated the excessive activation of autophagy in cells resulting from OGD; (C) Trehalose pre-treatment alleviated OGD-induced AMPK, p-AMPK, ULK1 and p-ULK1 protein expression and (D) Trehalose pre-treatment up-regulated protein expression of HKII, PFKP, PKM2, key rate-limiting enzymes of glycolysis

Note: *p<0.05 compared with the control group 
Oxyglucose deprivation has been shown to induce neuronal autophagy, which acts as an intracellular catabolic process. Damaged proteins and organelles are destroyed through lysosome-dependent pathways to restore homeostasis, but autophagy is a doubleedged sword in ischemic brain injury and its function is somewhat controversial. Although it has been reported that activation of autophagy attenuates cerebral infarction volume caused by local ischemia and reperfusion, it has also been found that excessive activation of autophagy is found to explain exacerbating brain injury caused by transient ischemia ${ }^{[16,17]}$. It has been reported that mitochondrial fusion protein 2 improves cerebral ischemia-reperfusion injury mainly by activating autophagy in primary cultured neurons ${ }^{[7]}$. However, some findings have shown that the autophagy inhibitors 3-MA and bafilomycin A1 can reduce infarct volume and the degree of brain edema in a rat model of cerebral ischemia by inhibiting autophagy ${ }^{[7]}$. We found that the expression of autophagy-related proteins LC3, beclin 1 and ATG5 was increased and the expression of p62 protein was decreased under OGD and immunofluorescence microscopy showed that LC3labeled autolysosomes were increased and it is generally accepted that the increase in autophagic flux is a strong evidence of autophagy activation ${ }^{[18]}$; meanwhile, autophagy inhibitors 3MA and ATG5 siRNA could inhibit OGD induced SH-SY5Y cell death, which is consistent with previous findings that inhibition of excessive activation of autophagy could rescue OGDinduced SH-SY5Y death ${ }^{[14]}$. Therefore, we suggest that OGD causes excessive activation of autophagy in SH-SY5Y cells which causes cell autophagic death. The difference in the protective role that this study plays for autophagy in previous reports may be due to the different cell lines used in the experiment and the context in which autophagy was induced.

In the pathogenesis of cerebral ischemic injury, irreversible damage to neural cells occurs due to the complete cessation of glucose and oxygen supply in the ischemic core area. Glucose serves as a major source of energy required for brain activity and its uptake/metabolic dysfunction is associated with the development of a variety of neurodegenerative disease ${ }^{[19]}$. Studies have found that brain tissue is highly susceptible to glucose metabolism disorders due to high energy requirements and high glucose consumption ${ }^{[20]}$. Therefore, ischemic cerebrovascular disease often causes cell death due to energy failure blood supply area. Therefore, improving glucose metabolism is important for the treatment of cerebral ischemic injury.
The results of this study showed that HKII, PFKP and PKM2 were down-regulated under OGD treatment and the ATP and pyruvate contents in SH-SY5Y cells were significantly reduced, suggesting that we could induce a decrease in the expression of key enzymes of glycolysis in SH-SY5Y cells by OGD leading to a decrease in ATP production caused by glycolytic dysfunction. Considering that OGD can cause both autophagic death and glycolytic dysfunction in SH-SY5Y cells, it provides us with research direction to explore the relationship between glycolysis and autophagic death. It has been reported that HKII controls the state of cellular energy homeostasis mediated by glycolysis and mammalian Target of Rapamycin Complex 1 (mTORC1). HKII detects binding and inhibits mTORC1 and enhances autophagy after glucose consumption ${ }^{[21]}$. Up regulation of PKM2 protein levels caused by pharmacological levels is involved in inhibiting the cellular autophagy pathway ${ }^{[22]}$.

PFKP is a rate-controlling enzyme of the glycolytic pathway and a recent study reported that PFKP significantly inhibited LC3-II and ATG5 expression and reduced starvation-mediated autophagy in oral squamous cell carcinoma ${ }^{[23]}$ and differently, PFKP down-regulation has also been reported to induce autophagy in cells ${ }^{[24]}$. There is a complex mutual regulation between autophagy and glycolysis, which still needs further study.

Both autophagy and glycolysis have been reported to be associated with regulation of glucose metabolism during cell starvation ${ }^{[25,26]}$. During cerebral ischemia/ reperfusion, Reactive Oxygen Species (ROS) overproduction causes oxidative damage to key proteins of glycolysis and ATP synthase, leading to decreased ATP causes many changes in brain function, such as altered membrane potential and increased intracellular $\mathrm{Ca}^{2+}$ levels, producing detrimental effects on cell function and survival ${ }^{[27]}$. In this experimental study, we found that the mechanism of OGD-induced glycolytic dysfunction may be the down-regulation of the expression of key rate-limiting enzymes of glycolysis. Ischemia and hypoxia had a strong inhibitory effect on the activities of key rate limiting enzymes HKII, PFKP and PKM2 ${ }^{[28]}$, resulting in the decrease of glycolysis rate and ATP production. AMPK is a well-established energy sensor that is usually allosterically activated by AMP and the increased ratio of AMP to ATP leads to AMPK phosphorylation, which triggers cellular autophagic death ${ }^{[8-10]}$. Combined with the results of this experiment, we speculate that OGD-induced 
glycolytic dysfunction may be caused by OGD-induced cellular oxidative stress, leading to oxidative damage of glycolytic key proteases and ATP synthase in cells, ultimately leading to cellular energy failure and then causing cellular autophagic death.

Trehalose is a non-reducing sugar composed of two glucose molecules with 1,1-glycosidic bonds, which as a safe and reliable natural sugar, plays an important role in maintaining the normal metabolic process of energy. Previous studies have reported that trehalose ameliorates oxidative stress-mediated reduction in mitochondrial membrane potential and decrease in ATP levels ${ }^{[29]}$. In this experiment, ATP production was significantly increased after trehalose pretreatment compared with the OGD group, suggesting that trehalose can change the intracellular energy status. However, the molecular target of trehalose has been difficult to determine for more than a decade. Trehalose as a therapeutic agent can easily cross the blood-brain barrier and improve cognitive dysfunction and behavioural characteristics in a rat model of transient global ischemia ${ }^{[14]}$. Previous studies have found that trehalose cannot only protect neurons from ischemic injury by alleviating the excessive activation of autophagy, but also inhibit cell death by inducing protective autophagy, which is different from the study of trehalose in protecting neuronal injury by inducing protective autophagy ${ }^{[30,16]}$. The data of this study showed that trehalose inhibited the excessive activation of lethal autophagy caused by ischemic injury and it was speculated that the mechanism may be that trehalose could reduce the inhibition of HKII, PFKP and PKM2, which are key rate-limiting enzymes of glycolysis by OGD, to increase the glycolytic rate and then maintain cellular energy metabolism homeostasis, inhibit AMPK phosphorylation and alleviate autophagic death. Unfortunately in humans, trehalose cannot be taken orally because it is degraded by trehalase present in the gastrointestinal tract. There are reports that trehalose can be given intravenously, but this requires registration of trehalose as an approved $\operatorname{drug}^{[31]}$. The amount of trehalose absorbed in human tissues is still unknown, which undoubtedly limits its potential clinical application.

In conclusion, trehalose can rescue the autophagic death of SH-SY5Y cells by improving OGD-induced glycolytic function and inhibiting the activation of AMPK signaling pathway. Therefore, alleviating glycolytic dysfunction as well as excessive activation of autophagy, may be a new potential therapeutic strategy for ischemic cerebrovascular disease.

\section{Funding:}

This article was supported by the National Natural Science Foundation of China (81771795), the Fundamental Research Business Expenses of Higher Education Institutions in Heilongjiang Province (2018-KYYWFMY-0002,2018-KYYWFMY-0053), the Mudanjiang Science and Technology Project (Z2016s0066,Z2008s065).

\section{Acknowledgment:}

Fengjiang Chen and Changhao Yin have contributed equally to this work and are considered as corresponding authors.

\section{Conflict of interests:}

The authors declared no conflicts of interest.

\section{REFERENCES}

1. Lu J, Qian HY, Liu LJ, Zhou BC, Xiao Y, Mao JN, et al. Mild hypothermia alleviates excessive autophagy and mitophagy in a rat model of asphyxial cardiac arrest. Neurol Sci 2014;35(11):1691-9.

2. Kim JH, Nam B, Choi YJ, Kim SY, Lee JE, Sung KJ, et al. Enhanced glycolysis supports cell survival in EGFR-mutant lung adenocarcinoma by inhibiting autophagy-mediated EGFR degradation. Cancer Res 2018;78(16):4482-96.

3. Chu Y, Chang Y, Lu W, Sheng X, Wang S, Xu H, et al. Regulation of autophagy by glycolysis in cancer. Cancer Manag Res 2020;12:13259.

4. Krüger U, Wang Y, Kumar S, Mandelkow EM. Autophagic degradation of tau in primary neurons and its enhancement by trehalose. Neurobiol Aging 2012;33(10):2291-305.

5. Zhang HS, Zhang ZG, Zhou Z, Du GY, Li H, Yu XY, et al. PKM2-mediated inhibition of autophagy facilitates Tat's inducing HIV-1 transactivation. Arch Biochem Biophys 2017;625:17-23.

6. Xie Z, Cao BQ, Wang $\mathrm{T}$, Lei Q, Kang $\mathrm{T}$, Ge CY, et al. LanCL1 attenuates ischemia-induced oxidative stress by Sirt3mediated preservation of mitochondrial function. Brain Res Bull 2018;142:216-23.

7. Castillo K, Nassif M, Valenzuela V, Rojas F, Matus S, Mercado $\mathrm{G}$, et al. Trehalose delays the progression of amyotrophic lateral sclerosis by enhancing autophagy in motoneurons. Autophagy 2013;9(9):1308-20.

8. Tan VP, Miyamoto S. HK2/hexokinase-II integrates glycolysis and autophagy to confer cellular protection. Autophagy 2015;11(6):963-4.

9. Peng C, Rao W, Zhang L, Gao F, Hui H, Wang $\mathrm{K}$, et al. Mitofusin 2 exerts a protective role in ischemia reperfusion injury through increasing autophagy. Cell Physiol Biochem 2018;46(6):2311-24.

10. Tu D, Gao Y, Yang R, Guan T, Hong JS, Gao HM. The pentose phosphate pathway regulates chronic neuroinflammation and dopaminergic neurodegeneration. J Neuroinflammation 2019;16(1):255.

11. Gao Z, Dlamini MB, Ge H, Jiang L, Geng C, Li Q, et al. ATF4mediated autophagy-dependent glycolysis plays an important role in attenuating apoptosis induced by $\mathrm{Cr}$ (VI) in A549 cells. Toxicol Lett 2020;331:178-87. 
12. Pagliassotti MJ, Estrada AL, Hudson WM, Wei Y, Wang D, Seals DR, et al. Trehalose supplementation reduces hepatic endoplasmic reticulum stress and inflammatory signaling in old mice. J Nutr Biochem 2017;45:15-23.

13. Marín-Hernández Á, Gallardo-Pérez JC, HernándezReséndiz I, Del Mazo-Monsalvo I, Robledo-Cadena DX, Moreno-Sánchez R, et al. Hypoglycemia enhances epithelialmesenchymal transition and invasiveness, and restrains the warburg phenotype, in hypoxic HeLa cell cultures and microspheroids. J Cell Physiol 2017;232(6):1346-59.

14. Shi D, Zhao D, Niu P, Zhu Y, Zhou J, Chen H. Glycolysis inhibition via mTOR suppression is a key step in cardamonininduced autophagy in SKOV3 cells. BMC Complement Alternat Med 2018;18(1):1-8.

15. Li Y, Luo Y, Luo T, Lu B, Wang C, Zhang Y, et al. Trehalose inhibits protein aggregation caused by transient ischemic insults through preservation of proteasome activity, not via induction of autophagy. Mol Neurobiol 2017;54(9):6857-69.

16. Köhrmann M, Schwab S. Treatment of stroke. Med Klin Intensivmed Notfmed 2017;112(8):666-7.

17. Tapia H, Koshland DE. Trehalose is a versatile and longlived chaperone for desiccation tolerance. Curr Biol 2014;24(23):2758-66.

18. Wei R, Mao L, Xu P, Zheng X, Hackman RM, Mackenzie GG, et al. Suppressing glucose metabolism with epigallocatechin3-gallate (EGCG) reduces breast cancer cell growth in preclinical models. Food Funct 2018;9(11):5682-96.

19. Tanji K, Miki Y, Maruyama A, Mimura J, Matsumiya T, Mori $\mathrm{F}$, et al. Trehalose intake induces chaperone molecules along with autophagy in a mouse model of Lewy body disease. Biochem Biophys Res Commun 2015;465(4):746-52.

20. Butterfield DA, Halliwell B. Oxidative stress, dysfunctional glucose metabolism and Alzheimer disease. Nat Rev Neurosci 2019;20(3):148-60.

21. Chen G, Liu H, Zhang Y, Liang J, Zhu Y, Zhang M, et al. Silencing PFKP inhibits starvation-induced autophagy, glycolysis and epithelial mesenchymal transition in oral squamous cell carcinoma. Exp Cell Res 2018;370(1):46-57.

22. Rusmini P, Cortese K, Crippa V, Cristofani R, Cicardi ME, Ferrari $\mathrm{V}$, et al. Trehalose induces autophagy via lysosomalmediated TFEB activation in models of motoneuron degeneration. Autophagy 2019;15(4):631-51.

23. Gao Z, Wang H, Zhang B, Wu X, Zhang Y, Ge P, et al. Trehalose inhibits $\mathrm{H} 2 \mathrm{O} 2$-induced autophagic death in dopaminergic SHSY5Y cells via mitigation of ROS-dependent endoplasmic reticulum stress and AMPK activation. Int $\mathrm{J}$ Med Sci 2018;15(10):1014.
24. Wang X, Lu S, He C, Wang C, Wang L, Piao M, et al. RSL3 induced autophagic death in glioma cells via causing glycolysis dysfunction. Biochem Biophys Res Commun 2019;518(3):590-7.

25. Pan BY, Li GY, Wu Y, Zhou ZS, Zhou M, Li C. Glucose utilization in the regulation of chitin synthesis in brown plant hopper. J Insect Sci 2019;19(5):3.

26. Soto-Heredero G, Gomez de las Heras MM, GabandéRodríguez E, Oller J, Mittelbrunn M. Glycolysis: A key player in the inflammatory response. FEBS J 2020;287(16):3350-69.

27. Phipps MS, Cronin CA. Management of acute ischemic stroke. BMJ 2020;368:16983.

28. Tang Q, Zheng G, Feng Z, Chen Y, Lou Y, Wang C, et al. Trehalose ameliorates oxidative stress-mediated mitochondrial dysfunction and ER stress via selective autophagy stimulation and autophagic flux restoration in osteoarthritis development. Cell Death Dis 2017;8(10):e3081.

29. Qin Y, Zheng B, Yang GS, Yang HJ, Zhou J, Yang Z, et al. Salvia miltiorrhiza-derived Sal-miR-58 induces autophagy and attenuates inflammation in vascular smooth muscle cells. Mol Ther Nucleic Acids 2020;21:492-511.

30. Shun CT, Lin SK, Hong CY, Lin CF, Liu CM. Sirtuin 6 modulates hypoxia-induced autophagy in nasal polyp fibroblasts via inhibition of glycolysis. Am J Rhinol Allergy 2016;30(3):179-85.

31. Nciri R, Desmoulin F, Allagui MS, Murat JC, Feki AE, Vincent $\mathrm{C}$, et al. Neuroprotective effects of chronic exposure of SH-SY5Y to low lithium concentration involve glycolysis stimulation, extracellular pyruvate accumulation and resistance to oxidative stress. Int $\mathrm{J}$ Neuropsychopharmacol 2013;16(2):365-76.

This is an open access article distributed under the terms of the Creative Commons Attribution-NonCommercial-ShareAlike 3.0 License, which allows others to remix, tweak, and build upon the work non-commercially, as long as the author is credited and the new creations are licensed under the identical terms

This article was originally published in a special issue, "Novel Therapeutic Approaches in Biomedicine and Pharmaceutical Sciences" Indian J Pharm Sci 2021:83(6) Spl Issue "262-269" 